\title{
O cuidador no contexto da hospitalizaçáo de crianças e adolescentes ${ }^{1}$
}

\author{
Rúbia Marques Pylóa, Maristela dos Guimarães Peixoto ${ }^{b}$, Kátia Maria Penido Bueno ${ }^{\mathrm{c}}$ \\ ${ }^{a}$ Centro Psíquico da Adolescência e Infância, Fundação Hospitalar do Estado de Minas Gerais - FHEMIG, \\ Belo Horizonte, MG, Brasil. \\ ${ }^{b}$ Residência Multiprofissional da Saúde da Criança, Hospital Municipal Odilon Behrens, \\ Belo Horizonte, MG, Brasil. \\ ${ }^{\mathrm{c}}$ Faculdade de Ciências Médicas de Minas Gerais - CMMG, Belo Horizonte, MG, Brasil.
}

\begin{abstract}
Resumo: Introdução: A permanência de um acompanhante em período integral com a criança e o adolescente hospitalizado, conforme determinado pelo Estatuto da Criança e do Adolescente (ECA), em 1990, é um fator essencial de humanização e acarreta uma modificação da estrutura formal de organização hospitalar. Objetivo: Conhecer as tendências atuais de estudo sobre cuidadores em ambiente de hospitalização de crianças e adolescentes, e identificar as ações direcionadas a este público. Metodologia: A busca foi realizada em setembro de 2014 na base de dados LILACS da Biblioteca Virtual em Saúde (BVS), utilizando-se a pesquisa por descritor de assunto, com os descritores "cuidadores" ou "acompanhantes de pacientes" ou "pais" e "hospitalização" ou "criança hospitalizada" ou "adolescente hospitalizado". Resultados/Discussão: Foram selecionados 11 artigos, com as seguintes características: 71\% eram da área da Enfermagem; 81\% foram realizados na Região Sul do Brasil; dez pesquisas foram qualitativas e uma, qualiquantitativa; oito estudos revelaram que a grande maioria dos participantes foi constituída pelas mães das crianças e dos adolescentes hospitalizados. Foram identificados alguns pontos em comum, levantados pelos cuidadores. Em $82 \%$ dos artigos da amostra, os cuidadores abordaram os vínculos estabelecidos por estes; em $73 \%$, destacaram a comunicação entre os acompanhantes e a equipe de saúde; em 64\%, apontaram as normas e rotinas hospitalares e, em $45 \%$ dos trabalhos, ressaltaram o aspecto da fé/religiosidade. Conclusões: Acredita-se que tais resultados poderão contribuir para a melhor compreensão das necessidades dos cuidadores de crianças e adolescentes hospitalizados. Dessa forma, este trabalho poderá auxiliar na construção de uma assistência hospitalar mais humanizada, melhorando a qualidade dos serviços.
\end{abstract}

Palavras-chave: Cuidadores, Pais, Hospitalização, Criança, Adolescente.

\section{The caregiver in the child/adolescent hospitalization context}

\begin{abstract}
Introduction: The full time permanence of a caregiver with hospitalized child and adolescents, as determined by the Brazilian Child and Adolescent Statute ("Estatuto de Criança e do Adolescente" - ECA), in 1990, is an essential humanization issue, which leads to a change in the formal structure of hospital organization. Objective: Knowing the current research trends on caregivers in the children and adolescent hospitalization environment and identifying actions directed to them. Methodology: the search was held in September 2014 in the LILACS database of Health Virtual Library ("Biblioteca Virtual em Saúde" - BVS), using the keyword search, with the keywords: "caregivers" or "patient's companions" or "parents" and "hospitalization" or "hospitalized child" or "hospitalized adolescent". Results/Discussion: We selected 11 papers; 71\% of those were from Nursing; 81\% were performed in the Southern Brazil; 10 were qualitative researches and 1 qualitative-quantitative; 8 studies revealed that most of the participants were the hospitalized child/adolescent's mother. The caregivers highlighted that in $82 \%$ of the
\end{abstract}

Autor para correspondência: Rúbia Marques Pyló, Centro Psíquico da Adolescência e Infância, Rua Padre Marinho, 150, Santa Efigênia, CEP 30140-040, Belo Horizonte, MG, Brasil, e-mail: rubiamarquespylo@yahoo.com.br

Recebido em Maio 19, 2015; $1^{\text {a }}$ Revisão em Ago. 13, 2015; Aceito em Set. 9, 2015. 
papers, the participants brought up the importance of the connection established by the caregivers; in $73 \%$ of them, the caregivers highlighted the communication between caregivers and the health staff; $64 \%$ pointed out the rules and hospital routines and $45 \%$ mentioned the faith and religiousness aspect. Conclusions: It is believed that these results may contribute to improve the comprehension of the hospitalized children and adolescents caregivers needs. Thus, this work will be able to help creating a more humanized hospital assistance and to improve the quality of services provided.

Keywords: Caregivers, Parents, Hospitalization, Child, Adolescent.

\section{Introdução}

Quando a criança ou o adolescente é internado em um hospital, com frequência, depara-se com uma situação nova e desconhecida, sendo submetido a tratamentos, exames e procedimentos muitas vezes invasivos e/ou dolorosos. Confronta-se com seu novo status de doente e passa a ocupar um espaço que o despersonaliza, pois está longe dos seus pertences e de sua individualidade (ALAMY, 2007). Constata-se que o desenvolvimento infantil pode ficar comprometido pelas experiências que a criança se vê obrigada a enfrentar (CHIATTONE, 2012a).

Neste contexto, a experiência mais difícil frente ao adoecimento infantil diz respeito ao afastamento das pessoas queridas e significativas à criança e ao adolescente, gerando sentimento de abandono. A separação da figura materna, por exemplo, numa situação de crise, em que a criança necessita de apoio e carinho, pode acarretar diversos problemas, dentre os quais depressão, perda de peso, apatia, inapetência, ansiedade, distúrbios do sono, atraso no desenvolvimento, regressão no processo de maturação psicoafetiva e falta de colaboração no tratamento (ALAMY, 2007). Dessa forma, é essencial que a família permaneça próxima à criança ou ao adolescente durante a hospitalização, sendo esta permanência um fator central na constituição do desenvolvimento infantil (CHIATTONE, 2012b).

Atendendo a esta necessidade, o Estatuto da Criança e do Adolescente (BRASIL, 1990, p. 3), no Título II, dos Direitos Fundamentais, Capítulo I, do Direito à Vida e à Saúde, artigo 12, determinou que:

Os estabelecimentos de atendimento à saúde deverão proporcionar condiçôes para a permanência em tempo integral de um dos pais ou responsável, nos casos de internação de criança ou adolescente [...].

Assim, foi criado, nas unidades hospitalares com atendimento à criança e ao adolescente, o alojamento conjunto.

Percebe-se que a permanência de um acompanhante em período integral com a criança ou o adolescente acarreta uma reestruturação das unidades pediátricas, alterando a estrutura formal da organização hospitalar (COLLET; ROCHA, 2004). Entretanto, nas práticas das autoras deste estudo, no atendimento à criança e ao adolescente em ambiente de internação, observa-se que este processo ainda está em construção, pois apesar dos avanços obtidos, identificam-se limitaçóes na integração dos acompanhantes no processo de tratamento.

Desta forma, este estudo teve como objetivo conhecer os trabalhos desenvolvidos no Brasil nos últimos dez anos junto a cuidadores de crianças/adolescentes hospitalizados, bem como auxiliar na identificação das tendências atuais de estudos e das açóes direcionadas a este público. A partir desta análise, espera-se reunir subsídios para o desenvolvimento de estratégias voltadas para o cuidador neste contexto, de forma que os serviços de saúde possam oferecer uma atenção mais resolutiva aos pacientes e sua família.

\section{Metodologia}

A busca foi realizada em setembro de 2014, na base de dados LILACS (Literatura Latino-Americana e do Caribe em Ciências da Saúde) da Biblioteca Virtual em Saúde (BVS), utilizando-se a pesquisa por descritor de assunto. Utilizaram-se os descritores "cuidadores" ou "acompanhantes de pacientes" ou "pais" e "hospitalização" ou "criança hospitalizada" ou "adolescente hospitalizado". Foram encontrados 87 artigos.

Destes, selecionaram-se os artigos que tinham como foco o cuidador; publicados em português; nos últimos dez anos, de setembro de 2004 a setembro de 2014, e que continham, no resumo, a especificidade do trabalho com cuidadores de crianças e/ou adolescentes em ambiente de internaçáo hospitalar.

Foram excluídos artigos com resumo indisponível na base de dados utilizada, com foco em testes e/ou avaliaçôes para cuidadores ou na percepção da equipe de saúde.

Após a análise dos critérios, dos 87 artigos inicialmente encontrados, foram selecionados 11 , cujos resultados principais serão apresentados e discutidos a seguir. Considerou-se, nessa apresentação dos 
resultados, a área de pesquisa, a regiáo de realização do estudo, o tipo de pesquisa - quantitativa ou qualitativa -, a amostra e também alguns temas em comum abordados com mais frequência nos trabalhos utilizados: os vínculos, a comunicação, as normas e rotinas, e a fé/religiosidade.

\section{Resultados}

Inicialmente, serão apresentados os resultados dos artigos seguindo-se os tópicos anteriormente citados e, posteriormente, seráo abordados os pontos em comum identificados.

Do total de 11 artigos encontrados, dez eram da área da Enfermagem e apenas o artigo de Quintana et al. (2011) é da Psicologia.

Quanto à regiấo de realização dos estudos, nove foram realizados na Regiáo Sul do Brasil e apenas dois em outras regiôes, sendo o de Sampaio et al. (2009) no Sudeste e o de Andraus et al. (2007), no Centro-Oeste brasileiros.

Em relação aos tipos de pesquisa, dez foram qualitativas e uma, qualiquantitativa. Das qualitativas, seis foram caracterizadas pelos autores como descritivas e exploratórias, uma como descritiva, exploratória e intervencionista, e uma somente como descritiva. Nos demais estudos qualitativos encontrados, não foi especificado o tipo de pesquisa.

Em relação à amostra, identificou-se, nos oito artigos que trouxeram essa informaçáo, que a maior parte dos participantes dos estudos era composta pelas mães das crianças ou dos adolescentes, com média de participação destas de $88 \%$. Segue a Tabela 1, com porcentagem de mães dentre os participantes dos estudos:

Referindo-se aos resultados apresentados por cada artigo, foram identificados alguns pontos em comum, levantados pelos cuidadores/acompanhantes, nomeando-os por "vínculos", "comunicação", "normas e rotinas" e "fé/religiosidade", que serão apresentados na Tabela 2, seguindo-se os comentários.

Um aspecto apontado foi a importância, para os cuidadores, dos vínculos que os mesmos estabelecem na situação de hospitalização da criança e do adolescente como um apoio bastante significativo no enfrentamento de suas dificuldades. Esta questáo é abordada por $82 \%$ dos artigos da amostra. Foram ressaltados os vínculos com os profissionais de saúde, com outros cuidadores na internação e com familiares, parentes, amigos e vizinhos.

Alguns autores (SILVA; WEGNER; PEDRO, 2012; GOMES et al., 2011; SAMPAIO et al., 2009; CENTA; MOREIRA; PINTO, 2004; ANDRAUS et al., 2007) apontaram os vínculos positivos estabelecidos entre a equipe de saúde e os cuidadores como um fator que interferiu na avaliaçáo da qualidade do atendimento oferecido no hospital, favorecendo o estabelecimento de uma relaçáo de confiança entre estes. Entretanto, alguns trabalhos (WEGNER; PEDRO, 2009; MILANESI et al., 2006) trouxeram a desconsideração dos cuidadores pela equipe de saúde como um aspecto negativo, o que acaba anulando a possibilidade de se ter a família como aliada na internação da criança e do adolescente.

Tabela 1. Porcentagem da quantidade de mães.

\begin{tabular}{|c|c|c|}
\hline & Referência & Porcentagem de mães dentre os participantes \\
\hline Artigo 1 & Xavier, Gomes e Salvador (2014) & Não trouxe esta informação. \\
\hline Artigo 2 & Silva, Wegner e Pedro (2012) & $\begin{array}{l}13 \text { participantes, sendo } 12 \text { mães e um pai - } 92 \% \text { constituído } \\
\text { por mães. }\end{array}$ \\
\hline Artigo 3 & Duarte, Zanini e Nedel (2012) & $\begin{array}{l}13 \text { participantes, sendo nove mães, dois pais e um casal }-77 \% \\
\text { mães. }\end{array}$ \\
\hline Artigo 4 & Quintana et al. (2011) & Dez participantes, todas mães $-100 \%$ mães. \\
\hline Artigo 5 & Gomes et al. (2011) & Não trouxe esta informação. \\
\hline Artigo 6 & Melo, Marcon e Uchimura (2010) & Não trouxe esta informação. \\
\hline Artigo 7 & Wegner e Pedro (2009) & Nove participantes, sendo oito mães e um pai - $89 \%$ mães. \\
\hline Artigo 8 & Sampaio et al. (2009) & 15 participantes, sendo todas mães - 100\% mães. \\
\hline Artigo 9 & Andraus et al. (2007) & 103 participantes, sendo $78 \%$ mães. \\
\hline Artigo 10 & Milanesi et al. (2006) & Oito participantes, sendo sete mães e um pai - $88 \%$ mães. \\
\hline \multirow[t]{2}{*}{ Artigo 11} & Centa, Moreira e Pinto (2004) & $\begin{array}{l}18 \text { participantes, sendo } 15 \text { mães, dois pais e uma avó - } 83 \% \\
\text { mães. }\end{array}$ \\
\hline & Média destes artigos & $88 \%$ dos participantes: mães. \\
\hline
\end{tabular}


Tabela 2. Divisão dos artigos por temas abordados.

- Artigo 2: Silva, Wegner e Pedro (2012).

- Artigo 3: Duarte, Zanini e Nedel (2012).

- Artigo 4: Quintana et al. (2011).

\section{Vínculos}

( $82 \%$ dos artigos da amostra abordaram esta questão)

\section{Comunicação}

(73\% dos artigos da amostra abordaram esta questão)

\section{Normas e rotinas}

(64\% dos artigos da amostra abordaram esta questão)

\section{Fé/Religiosidade}

(45\% dos artigos da amostra abordaram esta questão)
- Artigo 5: Gomes et al. (2011).

- Artigo 7: Wegner e Pedro (2009).

- Artigo 8: Sampaio et al. (2009).

- Artigo 9: Andraus et al. (2007).

- Artigo 10: Milanesi et al. (2006).

- Artigo 11: Centa, Moreira e Pinto (2004).

- Artigo 2: Silva, Wegner e Pedro (2012).

- Artigo 3: Duarte, Zanini e Nedel (2012).

- Artigo 6: Melo, Marcon e Uchimura (2010).

- Artigo 7: Wegner e Pedro (2009).

- Artigo 8: Sampaio et al. (2009).

- Artigo 9: Andraus et al. (2007).

- Artigo 10: Milanesi et al. (2006).

- Artigo 11: Centa, Moreira e Pinto (2004).

- Artigo 1: Xavier, Gomes e Salvador (2014).

- Artigo 3: Duarte, Zanini e Nedel (2012).

- Artigo 4: Quintana et al. (2011).

- Artigo 7: Wegner e Pedro (2009).

- Artigo 9: Andraus et al. (2007).

- Artigo 10: Milanesi et al. (2006).

- Artigo 11: Centa, Moreira e Pinto (2004).

- Artigo 3: Duarte, Zanini e Nedel (2012).

- Artigo 5: Gomes et al. (2011).

- Artigo 8: Sampaio et al. (2009).

- Artigo 9: Milanesi et al. (2006).

- Artigo 10: Centa, Moreira e Pinto (2004).
Ainda em relação ao estabelecimento de vínculos, foi ressaltada, em vários artigos (DUARTE; ZANINI; NEDEL, 2012; QUINTANA et al., 2011; GOMES et al., 2011; MILANESI et al., 2006; ANDRAUS et al., 2007), a importância dos vínculos entre os cuidadores no ambiente hospitalar, o que parece ser um instrumento de ajuda mútua, pela identificaçáo que ocorre entre estes. Tal contato parece minimizar o sentimento de solidão e desamparo no decorrer da hospitalização, propiciando cumplicidade, solidariedade e uma melhor aceitação da internação da criança ou do adolescente.

Ressaltou-se, ainda, (QUINTANA et al., 2011; GOMES et al., 2011; ANDRAUS et al., 2007) a importância dos familiares, parentes, amigos e vizinhos como um apoio que sustenta a ausência dos cuidadores principais no ambiente doméstico, além de ser relevante como um auxiliar nos cuidados com a criança hospitalizada e para o próprio cuidador. Destacou-se a relevância das visitas hospitalares e das ligaçóes telefônicas, pois propiciam esse contato.

Em relação ao aspecto "vínculos", Duarte, Zanini e Nedel (2012), Gomes et al. (2011) e Andraus et al. (2007) ressaltaram que é necessário que a instituição hospitalar, por meio da equipe de saúde, possa estimular a criaçáo e a manutenção de redes e vínculos que auxiliem no enfrentamento do cotidiano da hospitalização pelos acompanhantes.

Outro aspecto apontado pelos cuidadores foi a comunicação entre os acompanhantes e a equipe de saúde, sendo que tal questão foi ressaltada em $73 \%$ dos artigos. Duarte, Zanini e Nedel (2012) apontaram que o conhecimento sobre a doença é essencial para os pais, pois sentimentos de insegurança e culpa podem ser reduzidos com a necessidade de informaçáo sanada. Melo, Marcon e Uchimura (2010) afirmaram que a satisfação com as informaçóes recebidas é de extrema importância para diminuição da ansiedade e para favorecer a aceitação da doença e da hospitalização da criança pelos pais. Estes autores destacaram que, por outro lado, a comunicação insuficiente é um dos fatores que caracterizam a não humanização da assistência prestada, o que, segundo Milanesi et al. (2006), gera um estado de alerta constante dos acompanhantes, motivados pela preocupação.

Em diversos estudos (SILVA; WEGNER; PEDRO, 2012; MELO; MARCON; UCHIMURA, 2010; SAMPAIO et al., 2009; MILANESI et al., 2006; ANDRAUS et al., 2007), os participantes apontaram 
falhas nessa comunicação por parte da equipe, identificando: orientações e/ou informações recebidas superficiais, insuficientes; falta de esclarecimento dos procedimentos que são realizados; falta de conhecimento do diagnóstico médico, e comunicação verticalizada e desencontrada. Centa, Moreira e Pinto (2004) apontaram ainda que alguns pais relataram que receberam informaçóes, mas muitas vezes não entenderam o que foi explicado ou informado devido à tensão, ao estresse e ao cansaço.

Assim, os artigos indicaram a necessidade de uma maior atenção por parte dos profissionais de saúde para este aspecto, além de readequação da linguagem e da terminologia utilizadas.

Outro ponto levantado pelos cuidadores foi a questâo das normas e rotinas hospitalares, e a relação dos cuidadores com estas; tais questóes foram abordadas por $64 \%$ dos artigos da amostra. Centa, Moreira e Pinto (2004), Andraus et al. (2007), Wegner e Pedro (2009) e Quintana et al. (2011) apontaram, na visão do cuidador, a inflexibilidade das normas e regras hospitalares, com restriçóes impostas aos acompanhantes pela instituição, o que impactou na autonomia da família como cuidadora de seu filho. Xavier, Gomes e Salvador (2014) avaliaram que as normas e rotinas são consideradas pelos cuidadores como instrumentos importantes para organização do processo de trabalho, mas, segundo eles, em sua maioria, são elaboradas com o objetivo de beneficiar os profissionais em detrimento de crianças e familiares, o que, como constatou Milanesi et al. (2006), gera sofrimento psíquico. Avaliou-se que é importante que a equipe de saúde integre a família ao cuidado da criança, flexibilizando as normas, quando possível, de forma a permitir uma convivência mais harmoniosa com estas.

Foi ressaltado ainda, em $45 \%$ dos trabalhos, o aspecto da fé/religiosidade como uma estratégia significativa de enfrentamento da internação pelos participantes, o que também deve ser considerado pelos profissionais de saúde no contexto de internação. De acordo com Duarte, Zanini e Nedel (2012), a fé/religiosidade exerce um papel importante no equilíbrio emocional, na aceitaçáo e no enfrentamento da doença, sendo, segundo Sampaio et al. (2009), Milanesi et al. (2006) e Centa, Moreira e Pinto (2004), fonte de esperança, independentemente da religiáo ou do credo. Além disso, Gomes et al. (2011) afirmaram que a esperança na recuperação da criança ou do adolescente apresenta-se como um suporte que mantém a família e a fortalece emocionalmente.

Deve-se ressaltar ainda que todos os artigos apresentam estratégias para solucionar algumas das dificuldades apontadas, podendo-se citar: a importância da promoção em saúde, com o modelo de educação em saúde (WEGNER; PEDRO, 2009); a humanização da assistência, abordando-se o paciente em suas dimensôes biopsicossocial e espiritual (XAVIER; GOMES; SALVADOR, 2014; DUARTE; ZANINI; NEDEL, 2012; SAMPAIO et al., 2009; MILANESI et al., 2006); maior integração da família no processo terapêutico (MILANESI et al., 2006; CENTA; MOREIRA; PINTO, 2004), compreendendo-se que o familiar/ acompanhante também precisa de assistência da equipe de saúde (DUARTE; ZANINI; NEDEL, 2012; QUINTANA et al., 2011); a garantia do direito do acompanhante de ser adequadamente orientado e informado, estimulando-se a escuta, o diálogo e a compreensão (SILVA; WEGNER; PEDRO, 2012; DUARTE; ZANINI; NEDEL, 2012; MELO; MARCON; UCHIMURA, 2010; SAMPAIO et al., 2009; ANDRAUS et al., 2007; MILANESI et al., 2006; CENTA; MOREIRA; PINTO, 2004), além do reconhecimento e do fortalecimento das redes de apoio aos familiares/ acompanhantes (GOMES et al., 2011; ANDRAUS et al., 2007).

\section{Discussão}

Inicialmente, em relação à amostra de artigos utilizada neste trabalho, destacou-se a prevalência de publicaçôes na área de Enfermagem - dez em 11. Ressalta-se que a base de dados utilizada restringiu-se a LILACS, o que talvez explique a grande concentração de estudos dessa disciplina. Aponta-se a importância da abordagem do tema pelos profissionais da Enfermagem, pois são estes que mantêm um contato mais próximo e prolongado junto ao paciente e à sua família. Entretanto, sabe-se que existem publicaçóes sobre o tema em outras linhas de trabalho e a participação de pesquisadores de áreas diversas torna-se essencial para que o cuidado ao paciente e sua família seja efetivo, já que existe uma diversidade de profissionais que atuam junto a este público no ambiente hospitalar.

Além disso, observou-se que a maior parte dos estudos, nove em 11, foi realizada em hospitais da Região Sul do Brasil. Talvez essa discrepância também se relacione às limitaçóes desta pesquisa, que se ateve a apenas uma base de dados e a um número pequeno de artigos na composição da amostra.

Identificou-se ainda que, na amostra selecionada, não foi encontrado nenhum artigo que abordasse especificamente intervençôes junto aos cuidadores/ acompanhantes. Todos os trabalhos encontrados apresentavam pesquisas qualitativas/qualiquantitativas 
sobre a percepção do cuidador da internação hospitalar. As pesquisas de percepção são relevantes, pois, como apontam Silveira, Angelo e Martins (2008), a avaliação da estrutura e da dinâmica da família é muito importante para a promoção de ações adequadas às necessidades da unidade familiar. Entretanto, é necessário também desenvolver estratégias de cuidado à família na hospitalização, pois, como afirma Wanderbroocke (2005), esta consiste na principal fonte de apoio ao paciente, devendo também ser foco de intervençấo profissional. Citando Barros, Andrade e Siqueira (2013), os familiares cuidadores estão mais propensos a adoecer do que os demais membros da família, pois, além do contato direto com o paciente, o que já ocasiona sofrimento e mobiliza conteúdos relativos à morte, modifica-se sua vida de forma abrupta.

Dos 11 artigos da amostra, oito estudos apresentaram a informação que tiveram a mãe da criança ou do adolescente hospitalizado como a principal participante, com média de $88 \%$ dos participantes sendo máes. Este achado vai ao encontro do que é apontado por outros trabalhos, podendo-se citar pesquisa de Sabatés e Borba (2005), cuja amostra de 50 pais entrevistados em hospitalização de público infanto-juvenil era constituída por $82 \%$ de mães. Wanderbroocke (2005) afirma que o ato de cuidar de um familiar adoecido assume um perfil feminino e, na maior parte das vezes, restringe-se à família nuclear, sendo exercido por uma única pessoa. Barros, Andrade e Siqueira (2013) apontam que, quando apenas um familiar fica responsável pelos cuidados, este enfrenta sozinho as dificuldades e o sofrimento com o paciente, podendo desenvolver sintomas, como depressão, distúrbio do sono, isolamento social e afetivo, e falta de perspectiva de vida. Ressalta-se a importância da rede de apoio social, reforçando-se a contribuição dos serviços de saúde no fortalecimento destas redes, favorecendo $o$ acesso às mesmas e consistindo também em fonte de apoio ao cuidador.

Já em relação aos resultados dos artigos analisados, identificaram-se tanto aspectos que favorecem quanto aqueles que dificultam a integração dos cuidadores na assistência à criança e ao adolescente hospitalizado. Avalia-se que ocorreram progressos consideráveis, ressaltando-se a conquista deste direito na diminuição dos impactos da hospitalização na saúde dos pacientes. Atualmente, a humanização da assistência e o cuidado centrado na família são foco de atenção dos profissionais, devendo direcionar as práticas em saúde. Entretanto, percebe-se que ainda existem falhas nesse processo. Parece que existe um despreparo de alguns profissionais, refletindo-se na organizaçáo e na dinâmica dos serviços, acarretando problemas na comunicação, desinformação, falta de apoio e conflitos com o cuidador, gerando, assim, uma assistência fragmentada.

Sabatés e Borba (2005), em estudo com pais de crianças hospitalizadas, também apontam que estes não estão totalmente satisfeitos com as informações recebidas durante a hospitalização do filho, concluindo-se que não estão sendo atendidos em suas necessidades de informação. Collet e Rocha (2004), também em pesquisa com cuidadores em unidade pediátrica, encontraram que a equipe de Enfermagem não estabelece um diálogo com as mães para realizar uma negociação na divisão de trabalho, caracterizando-se uma relaçáo de dominação-subordinação e não de colaboração. Assim, concluem que é imprescindível reforçar a importância de atitudes de empatia nesta relação, de forma a criar um ambiente em que as mães se sintam seguras e fortalecidas. Oliveira, Dantas e Fonseca (2004) apontam que, apesar da constatação de que o alojamento conjunto pediátrico é relevante para a minimização do impacto da hospitalização, percebe-se que, algumas vezes, a infraestrutura dos hospitais conveniados com o Sistema Único de Saúde (SUS) dificulta a presença dos acompanhantes na internação.

Percebe-se, assim, que precisa haver um investimento na qualificação dos profissionais de saúde, integrando-se esta discussão nos currículos dos cursos da área e também na educação continuada e na capacitação dos profissionais. Além disso, identifica-se que os gestores precisam considerar o cuidador e suas necessidades ao planejar a estrutura e a dinâmica de atendimento hospitalar, favorecendo o acesso e a permanência deste com mais conforto e qualidade.

Identifica-se ainda a relevância de que sejam realizadas mais pesquisas na área que validem os investimentos na saúde do cuidador, gerando diminuiçáo dos custos e do tempo de internação, além da redução dos conflitos entre cuidadores e equipe, refletindo-se na melhoria da saúde dos profissionais e na maior satisfaçáo com o cuidado apontada por cuidadores e pacientes.

\section{Conclusão}

Avalia-se que este estudo apresenta algumas limitaçôes, podendo-se citar a utilização de apenas uma base de dados, com o objetivo de reduzir o número de artigos encontrados, possibilitando-se a análise. Além disso, os artigos da amostra se concentraram na área de Enfermagem, na Região Sul do país e 
apresentam, em sua maioria, pesquisas qualitativas de percepçáo do cuidador sobre a internação de crianças e adolescentes. Tais limitaçóes restringem a representatividade da amostra de artigos encontrados em relação à compreensão do contexto brasileiro de publicaçôes científicas recentes - últimos dez anos - nesta área.

Entretanto, por meio deste trabalho, foi possível identificar alguns fatores que favorecem ou dificultam a integração do cuidador no tratamento oferecido à criança e ao adolescente, sendo estes: o estabelecimento de vínculos com a equipe, com outros cuidadores e com familiares, parentes, amigos e vizinhos; a comunicação com a equipe de saúde; as normas e rotinas hospitalares, e a fé/religiosidade.

Assim, percebe-se que, a partir dos fatores identificados, foi possível atingir o objetivo do estudo de reunir subsídios para o desenvolvimento de estratégias voltadas para o cuidador neste contexto. Notou-se também a importância de se considerarem as demandas do cuidador ao se estruturar a rotina hospitalar, as normas e regras, além da necessidade de readequaçấo da estrutura física do hospital. Identificou-se também que é essencial possibilitar o estabelecimento e a manutenção das redes de apoio social e dos momentos em que os vínculos são reforçados, tais como por meio das visitas hospitalares e ligaçóes telefônicas, bem como o exercício da religiosidade.

Desta forma, acredita-se que serão realizados avanços em relação à construção de uma assistência hospitalar à criança e ao adolescente mais humanizada. Tais mudanças contribuirão para aprimorar a qualidade dos serviços, obtendo-se benefícios para o tratamento do paciente, com melhores resultados, maior satisfação deste e de seus familiares, e a melhoria do ambiente de trabalho para os profissionais de saúde.

\section{Referências}

ALAMY, S. Crianças hospitalizadas. In: ALAMY, S. Ensaios de psicologia hospitalar: a ausculta da alma. Belo Horizonte: Edição Independente, 2007. p. 117-135.

ANDRAUS, L. et al. Incidentes críticos segundo os familiares de crianças hospitalizadas. Revista Enfermagem UERJ, Rio de Janeiro, v. 15, n. 4, p. 574-579, 2007.

BARROS, S.; ANDRADE, M.; SIQUEIRA, F. Cuidar de um familiar com câncer: contribuições da terapia familiar sistêmica. Pensando Famílias, Porto Alegre, v. 17, n. 2, p. 96-110, 2013.

BRASIL. Lei no 8.069, de 13 de Julho de 1990. Dispôe sobre o Estatuto da Criança e Adolescente e dá outras providências. Diário Oficial [da] República Federativa do Brasil, Brasília, DF, 17 jul. 1990. Disponível em: <http:// www2.camara.leg.br/legin/fed/lei/1990/lei-8069-13-julho-1990-372211-normaatualizada-pl.pdf $>$. Acesso em: 04 nov. 2015.

CENTA, M.; MOREIRA, E.; PINTO, M. Experiência vivida pelas famílias de crianças hospitalizadas em uma unidade de terapia intensiva neonatal. Texto \& Contexto Enfermagem, Florianópolis, v. 13, n. 3, p. 444-451, 2004. http://dx.doi.org/10.1590/S010407072004000300015.

CHIATTONE, H. A criança e a morte. In: ANGERAMI-CAMON, V. A. et al. E a psicologia entrou no hospital. São Paulo: Cengage Learning, 2012a. p. 73-102.

CHIATTONE, H. A família e a morte da criança. In: ANGERAMI-CAMON, V. A. et al. (Org.). E a psicologia entrou no hospital. São Paulo: Cengage Learning, 2012b. p. 107-133.

COLLET, N.; ROCHA, S. Criança hospitalizada: máe e Enfermagem compartilhando o cuidado. Revista Latino-am Enfermagem, Ribeirăo Preto, v. 12, n. 2, p. 191-197, 2004. http://dx.doi.org/10.1590/S010411692004000200007.

DUARTE, M.; ZANINI, L.; NEDEL, M. O cotidiano dos pais de crianças com câncer e hospitalizadas. Revista Gaúcha de Enfermagem, Porto Alegre, v. 33, n. 3, p. 111-118, 2012. http://dx.doi.org/10.1590/S198314472012000300015 .

GOMES, G. et al. O apoio social ao familiar cuidador durante a internação hospitalar da criança. Revista Enfermagem UERJ, Rio de Janeiro, v. 19, n. 1, p. 64-69, 2011.

MELO, W.; MARCON, S.; UCHIMURA, T. A hospitalização de crianças na perspectiva de seus acompanhantes. Revista Enfermagem UERJ, Rio de Janeiro, v. 18, n. 4, p. 565-571, 2010.

MILANESI, K. et al. Sofrimento psíquico da família de crianças hospitalizadas. Revista Brasileira de Enfermagem, Brasília, v. 59, n. 6, p. 769-774, 2006. http://dx.doi. org/10.1590/S0034-71672006000600009.

OLIVEIRA, G.; DANTAS, F.; FONSECA, P. O impacto da hospitalizaçáo em crianças de 1 a 5 anos de idade. Revista da SBPH, Belo Horizonte, v. 7, n. 2, p. 37-54, 2004.

QUINTANA, A. et al. Lutos e lutas: reestruturações familiares diante do câncer em uma criança/adolescente. Psicologia Argumento, Curitiba, v. 29, n. 65, p. 143-154, 2011.

SABATÉS, A.; BORBA, R. As informaçōes recebidas pelos pais durante a hospitalização do filho. Revista Latino-am Enfermagem Ribeirão Preto, v. 13, n. 6, p. 968-973, 2005. http://dx.doi.org/10.1590/S010411692005000600008.

SAMPAIO, C. et al. Sentimentos dos acompanhantes de crianças submetidas a procedimentos cirúrgicos: vivências no perioperatório. Revista Mineira de Enfermagem, Belo Horizonte, v. 13, n. 4, p. 558-564, 2009. 
SILVA, T.; WEGNER, W.; PEDRO, E. Segurança da criança hospitalizada na UTI: compreendendo os eventos adversos sob a ótica do acompanhante. Revista Eletrônica de Enfermagem, Goiânia, v. 14, n. 2, p. 337-344, 2012.

SILVEIRA, A.; ANGELO, M.; MARTINS, S. Doença e hospitalização da criança: identificando as habilidades da família. Revista Enfermagem UERJ, Rio de Janeiro, v. 16, n. 2, p. 212-217. 2008.

XAVIER, D. M.; GOMES, G. C.; SALVADOR, M. S. O familiar cuidador durante a hospitalização da criança: convivendo com normas e rotinas. Escola Anna Nery, Rio de Janeiro, v. 18, n. 1, p. 68-74, 2014.

WANDERBROOCKE, A. Cuidando de um familiar com câncer. Psicologia Argumento, Curitiba, v. 23, n. 41, p. 17-23, 2005.

WEGNER, W.; PEDRO, E. Concepções de saúde sob a ótica de mulheres cuidadoras leigas, acompanhantes de crianças hospitalizadas. Revista Latino-am Enfermagem, Ribeirão Preto, v. 17, n. 1, p. 88-93, 2009. http://dx.doi. org/10.1590/S0104-11692009000100014.

\section{Contribuição dos Autores}

Maristela dos Guimarães Peixoto e Rúbia Marques Pyló: elaboração do estudo, análise dos dados e redação do artigo. Kátia Maria Penido Bueno: orientação em todas as etapas do trabalho e revisão do artigo. Todas as autoras aprovaram a versão final do texto.

\section{Notas}

${ }^{1}$ Este trabalho foi apresentado como pré-requisito para a obtenção do título de Especialista em Terapia Ocupacional na Saúde da Criança e do Adolescente do IPG (CMMG). 